\title{
Clinical utility of a computed tomography-based receiver operating characteristic curve model for the diagnosis of COVID-19
}

\author{
Xiang Li ${ }^{1 \#}$, Feng Yuan ${ }^{2 \#}$, Zhenguang Zhang ${ }^{2 \#}$, Juntao Yang ${ }^{3}$, Jing Zhang ${ }^{4}$, Zhipeng Li $^{5}$, Yan Peng ${ }^{6}$, \\ Yuanming Jiang ${ }^{2}$, Wenfang $\mathrm{Yi}^{2}$, Jiyao $\mathrm{Ma}^{2}$, Wei $\mathrm{Zhao}^{2}$, Bo $\mathrm{He}^{2,7}$, Li Wu ${ }^{2}$, Kunhua Wang ${ }^{7}$ \\ ${ }^{1}$ Department of Radiology, The 3rd Peoples' Hospital of Kunming, China; ${ }^{2}$ Medical Imaging Department, First Affiliated Hospital of Kunming \\ Medical University, Kunming Medical University, Kunming, China; ${ }^{3}$ Department of Radiology, Dali Bai Autonomous Prefecture People’s Hospital, \\ Dali, China; ${ }^{4}$ Department of Radiology, CT Room, People's Hospital of Yuxi City, Yuxi, China; ${ }^{5}$ Medical Imaging Department, Yunnan Provincial \\ Infectious Disease Hospital (Yunnan AIDS Care Center), Kunming, China; ${ }^{6}$ Department of Radiology, the First People's Hospital of Zhaotong, \\ Zhaotong, China; ${ }^{7}$ NHC Key Laboratory of Drug Addiction Medicine, First Affiliated Hospital of Kunming Medical University, Kunming Medical \\ University, Kunming, China \\ Contributions: (I) Conception and design: J Yang, J Zhang; (II) Administrative support: None; (III) Provision of study materials or patients: None; (IV) \\ Collection and assembly of data: J Yang, J Zhang; (V) Data analysis and interpretation: J Yang, J Zhang; (VI) Manuscript writing: All authors; (VII) \\ Final approval of manuscript: All authors. \\ "These authors contributed equally to this work as co-first authors. \\ Correspondence to: Bo He, MD. NHC Key Laboratory of Drug Addiction Medicine, Medical Imaging Department, First Affiliated Hospital of Kunming \\ Medical University, Kunming Medical University, Kunming 650031, China. Email: hebo_ydyy@qq.com; Li Wu, MD. Medical Imaging Department, \\ First Affiliated Hospital of Kunming Medical University, Kunming Medical University, Kunming 650031, China. Email: wuli211@163.com; \\ Kunhua Wang. NHC Key Laboratory of Drug Addiction Medicine, First Affiliated Hospital of Kunming Medical University, Kunming Medical \\ University, Kunming 650031, China. Email: wangkunhua_ydyy@163.com.
}

Background The outbreak of COVID-19 poses a major and urgent threat to global public health. CT findings associated with COVID-19 pneumonia from initial diagnosis until patient recovery. This study aimed to retrospectively analyze abnormal lung changes following initial computed tomography (CT) among patients with coronavirus disease 2019 (COVID-19) in Yunnan, and to evaluate the effectiveness of a chest CT-based model for the diagnosis of COVID-19.

Methods: One hundred and nine patients with COVID-19 pneumonia confirmed with the positive new coronavirus nucleic acid antibody who exhibited abnormal findings on initial CT were retrospectively analyzed. Thereafter, changes in the number, distribution, shape, and density of the lesions were observed. Further, the epidemiological, clinical, and CT imaging findings (+/-) were correlated. Following univariate and multivariate logistic regression analysis, receiver operating characteristic (ROC) curves were generated for significant factors, and models were established to evaluate the diagnostic ability of CT for COVID-19.

Results: Our results showed significant differences between patients with COVID-19 in epidemiological history (first, second, and third generation), clinical type (moderate, severe, and critical), and abnormal CT imaging characteristics $(+/-)(\mathrm{P}<0.05)$. Moreover, significant differences in abnormal CT imaging characteristics, including region, extent, and focus, were observed between the first generation and the other generations $(\mathrm{P}<0.05)$. For the diagnosis of COVID-19, the areas under the ROC curves for logistic regression models 1, 2, and 3 were 0.8016 (95\% CI: 0.6759-0.9274), 0.9132 (95\% CI: $0.8571-0.9693$ ), and 0.9758 (95\% CI: 0.9466-1), respectively.

Conclusions: The ROC curve regression model based on chest CT signs displayed a high diagnostic value for COVID-19.

Keywords! Novel coronavirus; pneumonia; computed tomography (CT); X-ray computer; ROC curve 
Submitted Nov 19, 2020. Accepted for publication Feb 03, 2021.

doi: 10.21037/apm-20-2603

View this article at: http://dx.doi.org/10.21037/apm-20-2603

\section{Introduction}

In December 2019, a cluster of cases of pneumonia with unknown cause was reported. Since then, the virus responsible for the outbreak, severe acute respiratory syndrome coronavirus 2 (SARS-CoV-2), has spread rapidly around the world, critically impacting human life and health, as well as the global economy $(1,2)$. On February 12, 2020, the World Health Organization (WHO) named the pneumonia induced by SARS-CoV-2 as coronavirus disease 2019 (COVID-19).

COVID-19 has strong infectivity and pathogenicity, characterized by human-to-human transmission, infection of medical staff, and rapid community spreading $(1,3,4)$. The main manifestations of COVID-19 include fever, dry cough, and fatigue, and the disease can be diagnosed by a positive nucleic acid test or virus gene sequencing (3). Respiratory droplets and contact transmission are considered to be main routes of transmission. At present, the diagnosis of COVID-19 is based on clinical symptoms, laboratory examination, and imaging examination, which require further confirmation through reverse transcription polymerase chain reaction (RT-PCR) viral nucleic acid detection or viral genome sequencing, with the former having been widely used. However, given the certain differences in the specificity and sensitivity of RT-PCR for the detection of COVID-19, which make it prone to producing false negative results, many experts have advocated the necessity of imaging examination.

Some studies have already reported on lung imaging features among patients with COVID-19 (5-7). Although a small number of researchers have summarized the characteristics of lung CT changes among hospitalized patients with COVID-19, imaging signs and criteria for the timely and accurate diagnosis of early-stage COVID-19, such as the nature, shape, and range of lesions, have not been described in detail $(8,9)$. Additionally, so far, $\mathrm{n}$ no study has focused on the ability of CT imaging to predict clinical classification and generation mentioned in the methods of COVID-19. Therefore, in the present study, we conducted a retrospective analysis of patients with COVID-19 who displayed abnormal lung CT findings on admission in order to identify more objective and accurate quantitative imaging parameters that could determine the nature of COVID-19 lesions and guide clinical strategies. It is hoped that this study will provide a reference for the clinical evaluation of curative effect and prognosis among patients with COVID-19.

We present the following article in accordance with the MDAR checklist (available at http://dx.doi.org/10.21037/ apm-20-2603).

\section{Methods}

All procedures performed in this study involving human participants were in accordance with the Declaration of Helsinki (as revised in 2013). This retrospective study was approved by the institutional review board of the First Affiliated Hospital of Kunming Medical University, Kunming Medical University, Kunming, China. Because of the retrospective nature of the research, the requirement for written informed consent was waived.

\section{General information}

As of 24:00 on March 14, 2020, 174 cases of COVID-19 had been confirmed in Yunnan; of them, 2 cases had died and the remaining 172 cases had recovered and been discharged. The inclusion criteria for patients in this study were: (I) confirmed with COVID-19 according to the diagnostic criteria in the Guidelines on the Novel Coronavirus-Infected Pneumonia Diagnosis and Treatment (1st to 7 th editions), issued by the National Health Commission of the People's Republic of China; (II) completed an initial chest CT examination within 3 days of being diagnosed with COVID-19; (III) complete clinical and laboratory examination data; and (IV) images in Digital Imaging and Communications in Medicine (DICOM) format with an image thickness of $\leq 2 \mathrm{~mm}$. Ultimately, 143 cases were included, among which 34 and 109 cases had normal and abnormal initial CT examination results, respectively. Among the 109 patients with abnormal initial CT results: 54 and 55 patients were males and females, respectively, with an age range of 3-78 years (mean age, $46 \pm 16.8$ years); 84 cases had a history of travel to or residence in Hubei province; and 20 cases had a history of close contact with confirmed cases (mainly relatives). Based 
on the epidemiological history of patients with COVID-19 in Yunnan, the first generation was defined those who had a history of travel to or residence in Hubei before disease onset and developed symptoms of COVID-19 after arriving in Yunnan; the second generation was defined as those who had no history of travel to Hubei before disease onset but had close contact with first-generation patients; the third generation was defined as those who had close contact with second-generation patients. Based on the COVID-19 diagnosis and treatment specifications, 87, 17, and 5 of the 109 patients were moderate, severe, and critical type, respectively. The main manifestations of COVID-19 included fever and respiratory-related symptoms.

\section{Case definition}

The diagnostic criteria and clinical classification of COVID-19 were based on the relevant contents presented in the Guidelines on the Novel Coronavirus-Infected Pneumonia Diagnosis and Treatment (1st to 7 th editions), issued by the National Health Commission of the People's Republic of China.

\section{Diagnostic criteria}

The epidemiological history and clinical manifestations were analyzed comprehensively:

Epidemiological history: (I) a history of travel to or residence in Wuhan and the surrounding areas or other infected communities within the 14 days before disease onset; (II) a history of contact with novel coronavirusinfected individuals (positive for nucleic acid detection) within the 14 days before disease onset; (III) a history of contact with patients with fever or respiratory symptoms from Wuhan and the surrounding areas or from infected communities within the 14 days before disease onset; and (IV) the epidemic of clusters. Clinical manifestations: (I) fever and/or respiratory symptoms; (II) the aforementioned imaging features of pneumonia; and (III) a normal or decreased white blood cell count or a decreased lymphocyte count in the early stages of the disease.

Individuals were suspected to have been infected when they satisfied: any item under epidemiological history and any 2 items under clinical manifestations; or, when no clear epidemiological history could be established, all 3 items under clinical manifestations. Suspected cases needed to satisfy 1 of the following etiological criteria: (I) Novel Coronavirus nucleic acid was tested positive in respiratory tract or hematological specimens by real-time fluorescencebased RT-PCR; and (II) viral genome sequencing results of respiratory tract or hematological specimens that were highly homologous to the known novel coronavirus. Patients who satisfied the following 2 criteria were included: (I) suspected cases of COVID-19; and (II) those with respiratory tract specimens that tested positive for novel coronavirus nucleic acid using real-time fluorescence-based RT-PCR.

\section{Clinical classification}

Mild type: mild clinical symptoms with no signs of pneumonia on imaging. Moderate type: fever, respiratory tract symptoms, and abnormal imaging findings. Severe type: satisfying any of the following criteria: (I) respiratory rate $(\mathrm{RR}) \geq 30$ times/minute suggesting respiratory distress; (II) oxygen saturation $\leq 93 \%$ at rest; or (III) arterial partial pressure of oxygen $\left(\mathrm{PaO}_{2}\right)$ /fraction of inspiration $\mathrm{O}_{2}\left(\mathrm{FiO}_{2}\right)$ $\leq 300 \mathrm{mmHg}(1 \mathrm{mmHg}=0.133 \mathrm{kPa})$. Critical type: satisfying any of the following conditions: (I) respiratory failure and need for mechanical ventilation; (II) shock; or (III) requiring intensive care unit monitoring and treatment Combined with other organ failure.

\section{Imaging examination and methods}

Patients underwent initial lung CT examination at medical institutions of all levels across Yunnan. Before and after the examination, the CT room was strictly disinfected by technicians using first-class protective equipment. Spiral CT scanners ( $\geq 16$ detector rows; Philips, GE, Siemens or United Imaging, etc.) were used with the following scanning parameters: tube voltage, $100-120 \mathrm{kV}$; tube current, 100-300 mAs; slice thickness, $5 \mathrm{~mm}$; thickness, 1-2 mm; interval, $0.7-1 \mathrm{~mm}$; and scanning range, from the base to the apex of the lungs.

Images were independently analyzed by 2 physicians who are considered experts in the field of chest imaging diagnosis. Disagreements between the 2 physicians were resolved through discussions mediated by a third highly qualified physician to obtain a consensus. Image analysis took into account the following: (I) lesion number: single or multiple; (II) lesion density: pure ground-glass opacity (GGO, is defined as hazy opacity that does not obscure underlying bronchial structures or pulmonary vessels at), pure consolidation (defined as an area with increased opacity, unclear vascular bundle, and a visible 


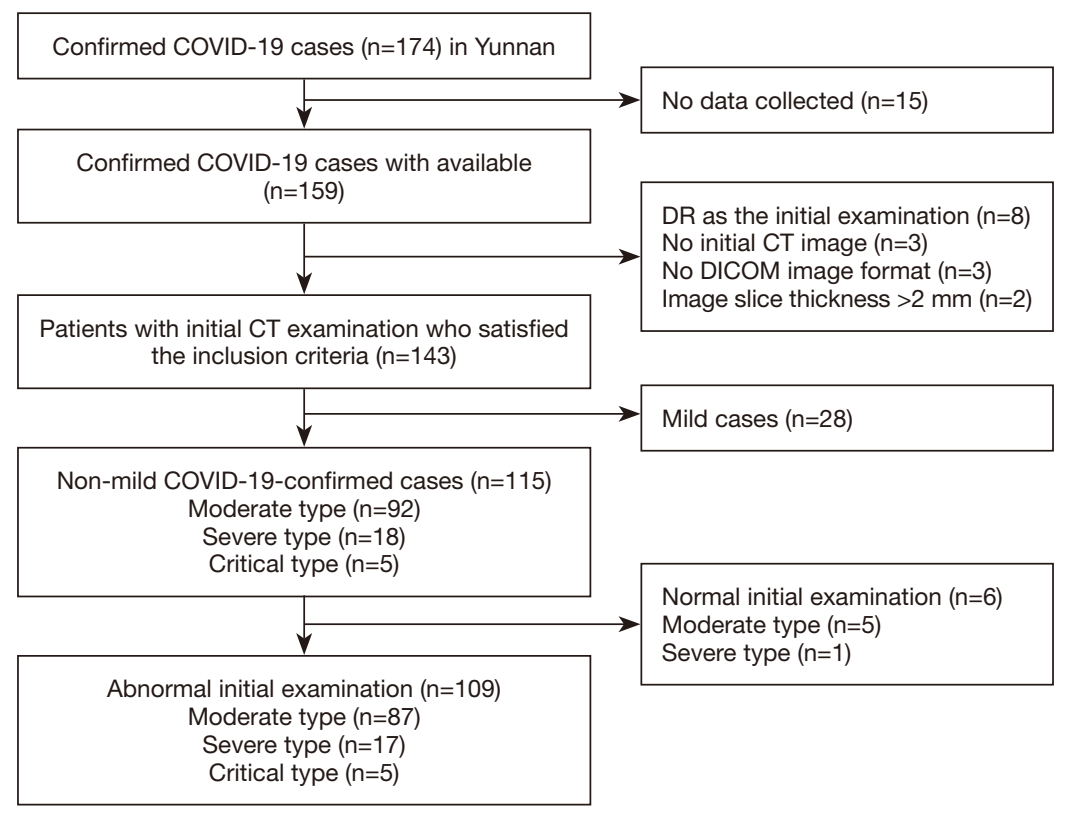

Figure 1 Flow chart for study inclusion and exclusion.

air bronchogram sign), or mixed opacity; (III) lesion range: peripheral involvement (subpleural or peripheral regions) or central involvement (lesions located in the lung segments or upper lung field); (IV) the number of lung segments involved (out of 18 segments); (V) lesion shape: round, wedge, or irregular; (VI) lesions associated with vascularity: visible of vessel thickening in pure GGO or mixed lesions and visible of multiple vascular structures converging toward the lesions (i.e., vascular convergence); (VII) the relationship between lesions and pleura: adjacent to the interlobular or visceral pleura of the chest wall; (VIII) interlobular pleural changes caused by lesions close to the interlobular pleura: interlobular pleural traction and displacement, and visible of interlobular septal and pleural thickening between the interlobular pleura; (IX) lesions near the visceral pleura: subpleural lucency shadow and visible of interlobular septal thickening and traction, visceral pleural thickening, or subpleural curvilinear shadow between the lesions and the visceral pleura; $(\mathrm{X})$ internal/peripheral features of the lesions: crazy-paving sign, halo sign, banyan crown sign, and bronchiectasis; (XI) mediastinal lymph node enlargement: transverse diameter $\geq 1 \mathrm{~cm}$; and (XII) pleural effusion.

\section{Statistical analysis}

ArcGIS 10.6 (2017 Esri.) was used to generate an image of the endemic distribution of confirmed COVID-19 cases in Yunnan province, and Microsoft Excel 2016 was used to establish a database. Data were expressed as the number of cases and constituent ratio [n (\%)] and were analyzed using the Chi-square test or Fisher's exact probability test. All statistical analyses were performed using SAS software. Receiver operating characteristic (ROC) curves were also generated, following which the area under the ROC curve (AUC) was calculated. All statistical analyses were 2-sided, with statistical significance indicated by $\mathrm{P}<0.05$.

\section{Results}

\section{General information (Table S1)}

In Yunnan, 174 patients from 46 medical institutions were confirmed to have COVID-19 from January 21.2020 to March 14, 2020. Based on the inclusion criteria, 109 patients were ultimately included in the present analysis (Figure 1). Among the 109 eligible COVID-19 patients, $54(49.54 \%)$ and $55(50.46 \%)$ were males and females, respectively. The patients ranged in age from 3-78 years (median age: 46 years); 22 cases $(20.18 \%)$ were $\leq 29$ years old, 16 cases (14.68\%) were between 30 and 39 years old, 22 cases $(20.18 \%)$ were between 40 and 49 years old, 18 cases $(16.51 \%)$ were between 50 and 59 years old, 22 cases $(20.18 \%)$ were between 60 and 69 years old, and 9 


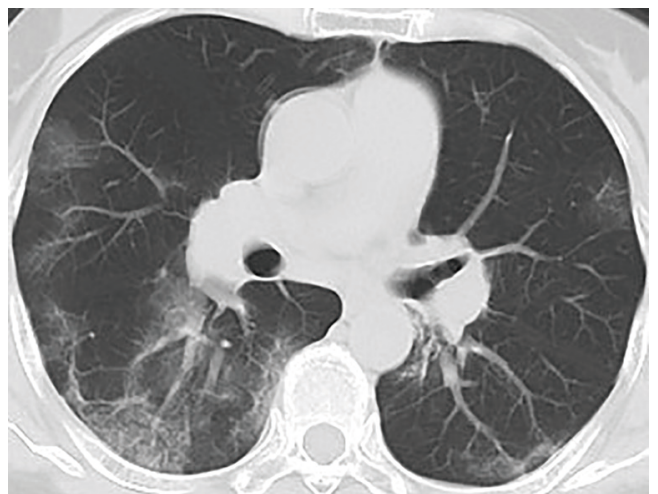

Figure 2 Multiple round-, wedge-, and irregular-shaped pure ground-glass opacity lesions in both lungs showing an enhancement shadow with a cloud-like density and visible bronchi and blood vessels.

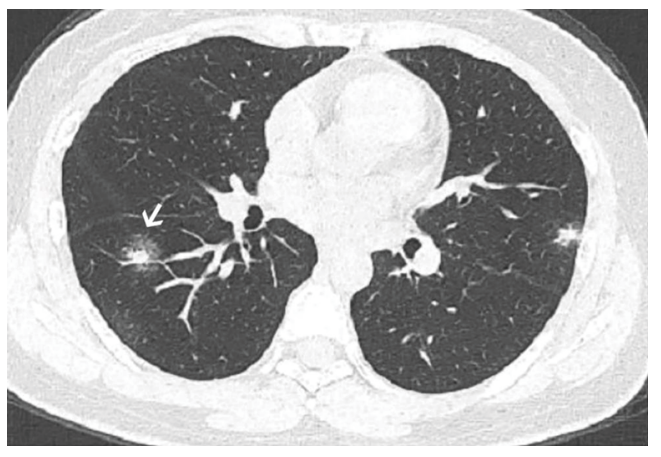

Figure 3 Mixed ground-glass opacity and consolidation lesions in the dorsal segment of the inferior lobe of the right lung (arrow) showing increased density at the center of the lesion (consolidation) and decreased density at the periphery of the lesion (ground-glass opacity).
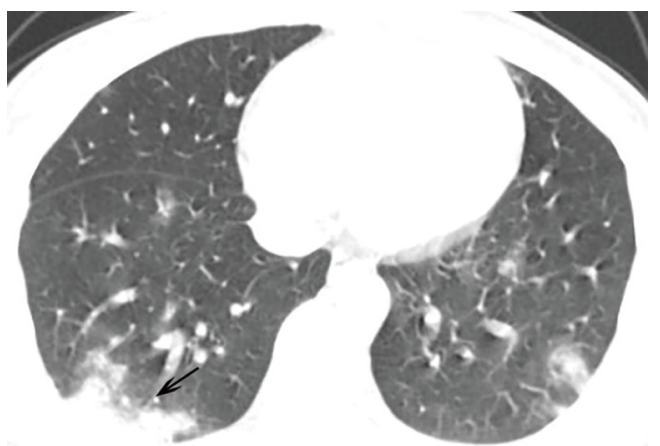

Figure 4 Pure consolidation lesions (arrow) near the chest wall in the posterior basal segment of the inferior lobe of the right lung showing increased density and an unclear vascular bundle. cases $(8.26 \%)$ were $\geq 70$ years old. Of the patients, $53.21 \%$ came from Hubei, $39.45 \%$ came from Yunnan, and the remainder came from Hunan $(2.75 \%)$, Anhui $(1.83 \%)$, Zhejiang (0.92\%), Guangdong (0.92\%), and Jiangxi (0.92\%). In terms of occupational status, retirees accounted for the largest proportion of patients (19.27\%), followed by office workers $(11.01 \%)$, the self-employed $(9.17 \%)$, and farmers $(8.26 \%)$, with only 1 patient being medical staff from Hubei. Regarding travel history, 51 cases were visitors by public transport $(38.53 \%)$ or had self-driven $(8.26 \%)$ from Wuhan, 25 cases (22.94\%) had returned to Yunnan after traveling to Wuhan, 18 cases $(16.51 \%)$ had a history of close contact with confirmed COVID-19 patients, and 11 cases had traveled via Wuhan $(8.26 \%)$ or had returned to Yunnan after a business trip $(0.92 \%)$ and leisure trip $(0.92 \%)$ to Wuhan (Table S1). Of the 109 patients, 84 patients were first generation, 20 were second generation, and 2 were third generation, with 3 patients having no clear epidemiological history.

\section{Initial chest computed tomography (CT) findings}

Upon initial chest CT examination, 12 patients (11.01\%) showed solitary lung lesions, and 97 (88.99\%) showed multiple lung lesions. The lesions observed were mainly round, wedge-shaped, or irregular-shaped. Table S2 details the location of the lesions. A total of 97 patients (88.99\%) had pure GGO lesions, as suggested by an enhancement shadow with cloud-like density and visible of the bronchi and blood vessels (Figure 2); 51 patients (46.79\%) had lesions with mixed GGO and consolidation with uneven density, light ground-glass changes, and consolidation shadow (Figure 3) (Table S2); and 16 patients (14.68\%) had pure consolidation (Figure 4) with a morphology similar to GGO, occasionally irregular shape, and a visible air bronchogram sign. Furthermore, 104 cases $(95.41 \%)$ had peripheral/subpleural lesions, while 45 cases $(41.28 \%)$ had lesions near the lung hilm (around the segmental and lobar bronchi) (Figure 5). Ninety-five cases $(87.16 \%)$ had visceral subpleural lesions of the chest wall (Figure 6), among which 63 cases $(57.8 \%)$ had subpleural lucency shadow (Figure $6 A), 62(56.88 \%)$ had interlobular septal thickening between the lesions and pleura (Figure 6B), 58 (53.21\%) had visceral pleural traction or thickening (Figure 6C), and 29 (26.61\%) had a subpleural line (Figure 6D). Moreover, 63 cases $(57.8 \%)$ had lesions near the interlobular pleura, including $52(47.71 \%)$ complicated by interlobular pleural traction and displacement (Figure $7 A$ ), 30 (27.52\%) with interlobular 


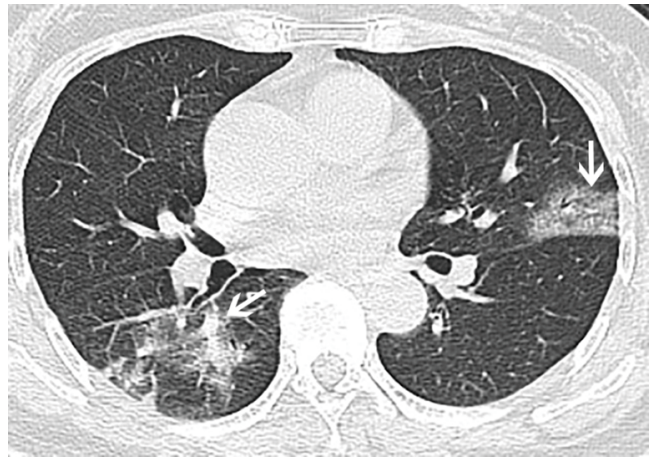

Figure 5 Upper lingual segment lesion in the superior lobe of the left lung located in the subpleural region of the lung periphery (arrow), and lesions near the lung hilar region (solid arrow) in the inferior lobe of the right lung, except those in the subpleural region of the lung periphery. septal thickening near the interlobular pleura (Figure $7 B)$, and $45(41.28 \%)$ complicated by interlobular pleural thickening (Figure 7C). Blood vessel thickening (79.82\%) (Figure $8 A$ ), and the vascular convergence sign $(59.63 \%)$ (Figure $8 B$ ), halo sign (50.46\%) (Figure 9A), banyan crown sign $(32.11 \%)$ (Figure 9B), and crazy-paving sign (29.36\%) (Figure 9C) were also observed, with a small number of patients also displaying bronchiectasis, mediastinal lymph node enlargement, and pleural effusion (Table S2).

\section{CT imaging analysis}

Based on the patients' baseline data and CT imaging results described in the methods statistical analysis was conducted on the patients' epidemiological history (first,
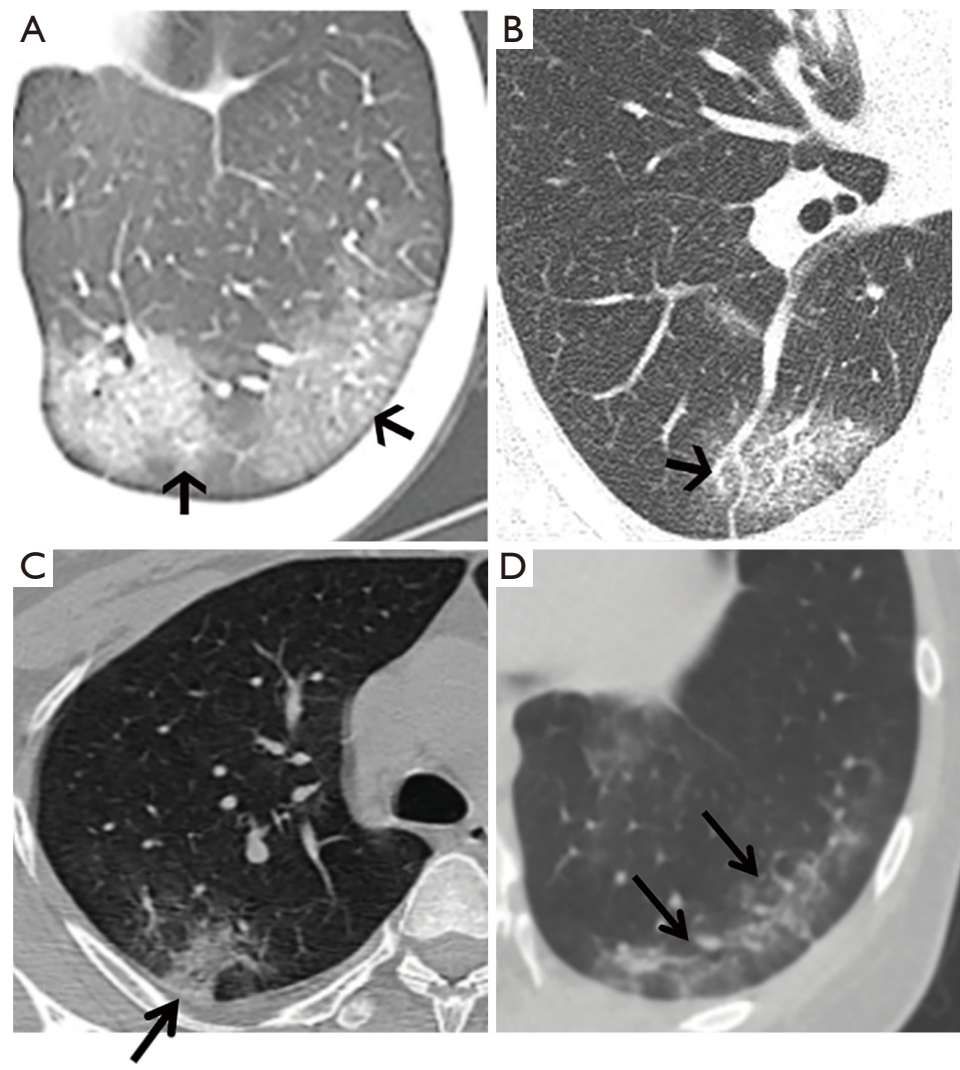

Figure 6 Lesions near the visceral pleura of the chest wall associated with changes. (A) Multiple patchy ground-glass opacities (GGOs) at the posterior basal segment of the inferior lobe of the left lung associated with subpleural lucency shadow (triangular arrow). (B) Multiple patchy GGOs associated with lobular septal thickening (solid arrow) at the posterior basal segment of the inferior lobe of the right lung, manifesting as a streak-like shadow perpendicular to the visceral pleura. (C) GGO in the posterior segment of the superior lobe of the right lung associated with visceral pleural thickening and traction (arrow). (D) Multiple patchy GGOs at the posterior and lateral basal segment of the inferior lobe of the left lung, accompanied by a subpleural curvilinear opacity (dovetail arrow), showing a strip-like hyperdense shadow parallel to the pleural direction. 

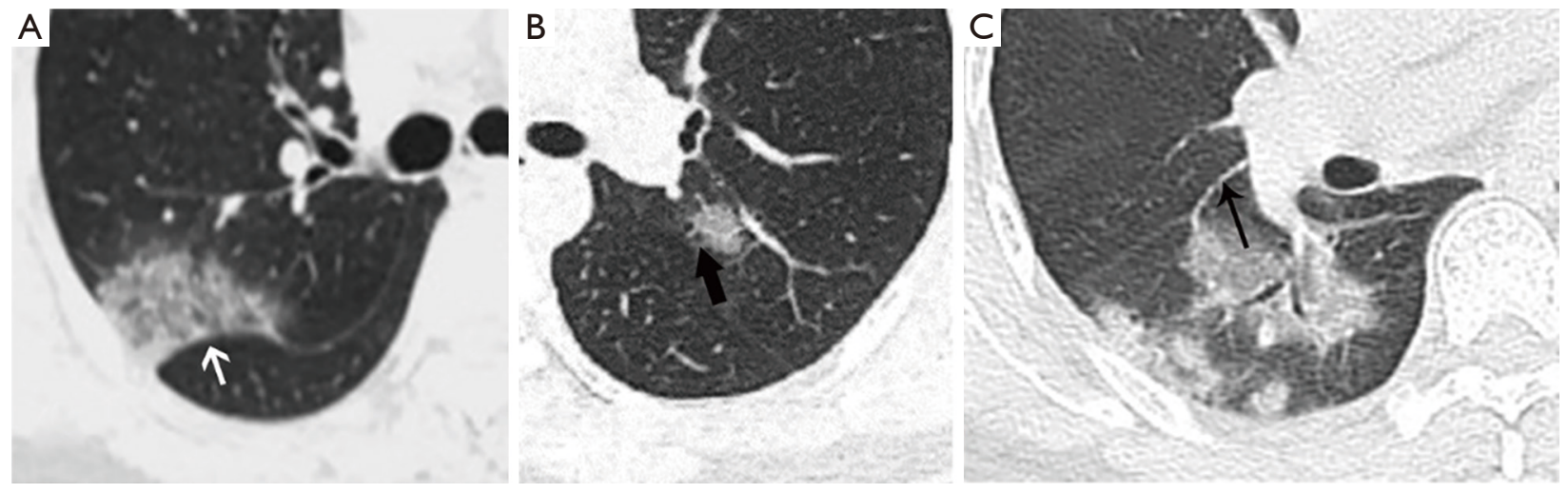

Figure 7 Lesions near the interlobar pleura associated with changes. (A) Posterior basal segment lesions in the superior lobe of the right lung located at the anterior edge of the right oblique fissure pleura showing obvious traction and displacement in the oblique fissure pleura (triangular arrow). (B) Ground-glass opacity (GGO) in the posterior segment of the superior lobe apex of the left lung, close to the left oblique fissure pleura, with visible interlobular septal thickening, manifesting as a stripe-like hyperdense shadow (solid arrow) perpendicular to the oblique fissure pleura. (C) Multiple GGOs in the dorsal segment of the inferior lobe of the right lung and lesions near the rightoblique fissure pleura, resulting in oblique fissure pleural thickening (arrow).
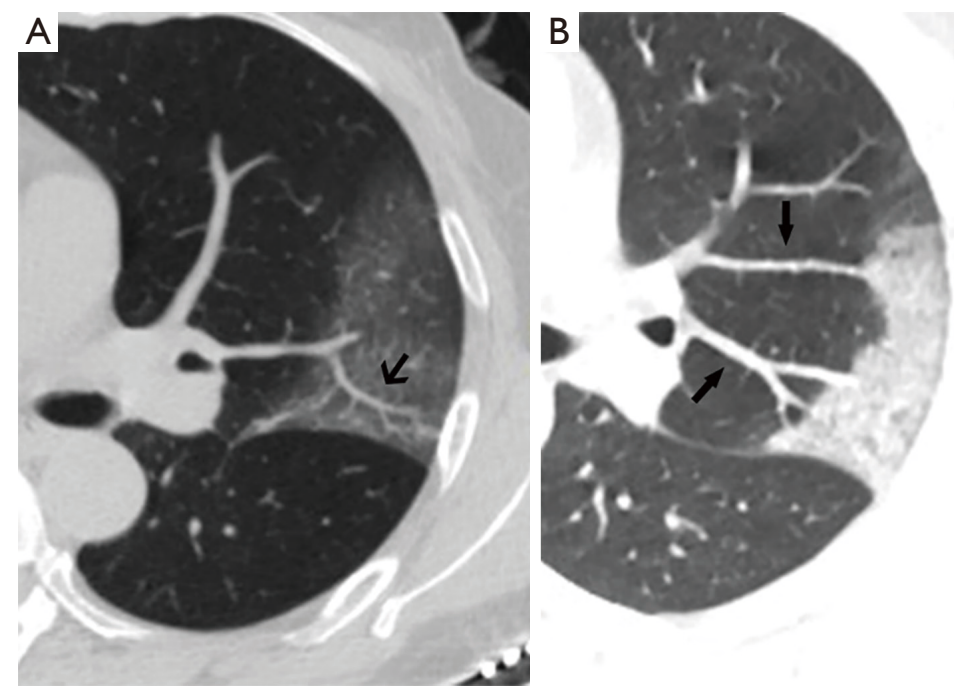

Figure 8 Lesions associated with vascular changes. (A) Ground-glass opacity (GGO) in the posterior segment of the superior lobe apex of the left lung associated with blood vessel thickening (triangular arrow) and (B) lesions in the posterior basal segment of the superior lobe of the left lung exhibiting a vascular convergence sign formed by the convergence of multiple blood vessels toward the lesions (solid arrow).

second, and third generations) and the 45 factors (covering the nature, range, shape, and location of the lesions) related to qualitative abnormal CT imaging signs " $(-/+)$ ", including pure GGO (-/+) and consolidation (-/+) lesions, mixed GGO and consolidation lesions $(-/+)$, peripheral and subpleural lesions $(-/+)$, lesions near the interlobar pleura $(-/+)$, lesions near the chest wall associated with blood vessel thickening $(-/+)$, interlobular septal thickening
$(-/+)$, and subpleural curvilinear shadow (-/+). Our analysis found significant differences in the constituent ratios for lesion location (the medial basal segment of the inferior lobe of the right lung), the presence of lesions near the chest wall accompanied by interlobular septal thickening and a subpleural curvilinear shadow, and lesions near the interlobular pleura accompanied by interlobular pleural traction and displacement according to epidemiological 

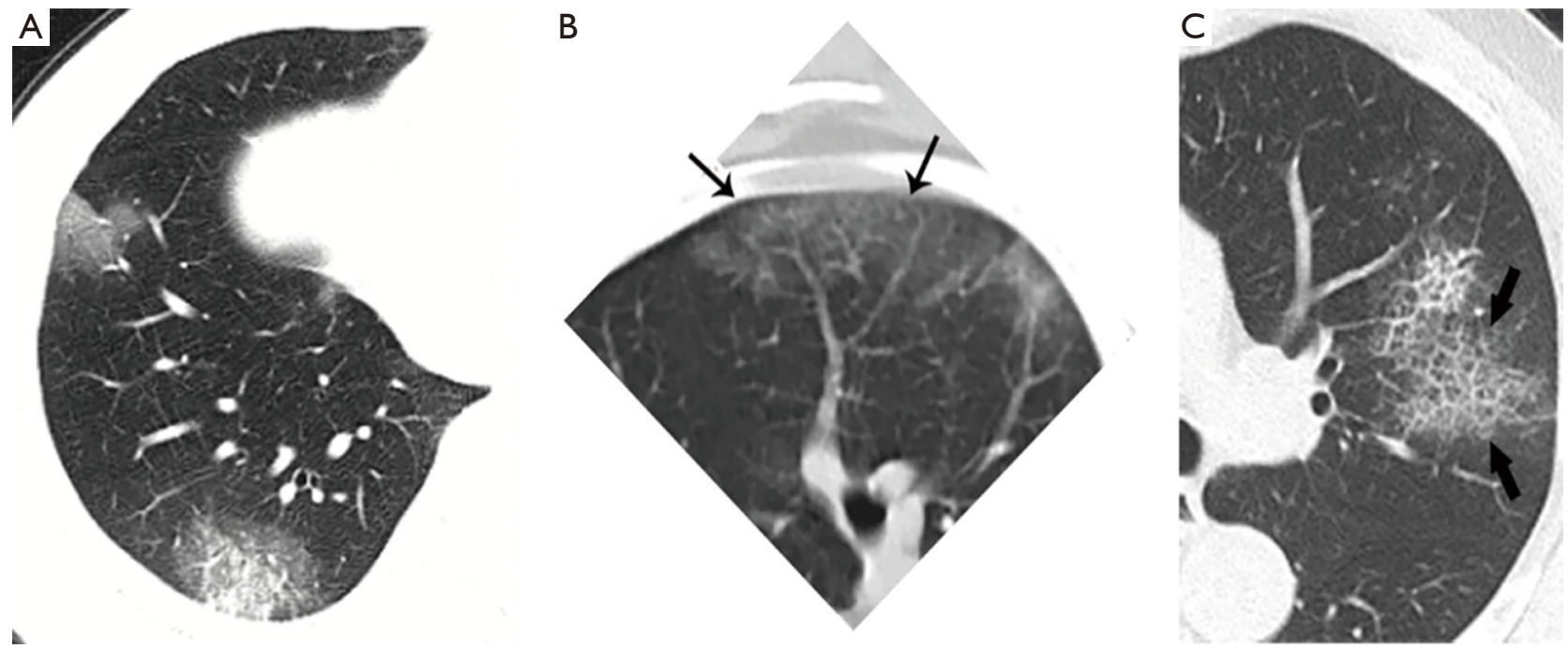

Figure 9 Other associated signs. (A) Ground-glass opacity (GGO) lesions near the pleura in the posterior basal segment of the inferior lobe of the right lung, with a visible lighter opacity shadow surrounding the lesions, known as the halo sign (solid arrow). (B) Multiple GGO lesions in the anterior basal segment of the superior lobe of the left lung, located in the peripheral region of the lung, with a wedge- and fan-shaped distribution and multiple visible blood vessels entering the lesions, resembling a banyan crown (arrow). (C) GGO lesions at the posterior segment of the superior lobe apex of the left lung, with visible grid-shaped or small honeycomb-like thickening of the interlobular septa, presenting as paving stone-like changes (solid arrow).

history $(\mathrm{P}<0.05)$ (Table S3).

Furthermore, stratified analysis of first- and non-first generation (i.e., second and third generation) patients revealed significant differences in abnormal CT imaging signs between first- and second-generation patients with regard to lesion location (the superior lingular segment of the superior lobe of the left lung), lesion range (near the lung hilum and around the segmental and lobar bronchus), lesions near the chest wall associated with interlobular septal thickening, lesions near the interlobular pleura associated with interlobular pleural thickening, interlobular pleural traction and displacement, and interlobular septal thickening of lesions near the interlobular pleura $(\mathrm{P}<0.05)$ (Table S4).

Furthermore, statistical analysis on the clinical classification of the 109 patients (moderate, severe, and critical type) based on qualitative abnormal CT imaging signs " $(-/+)$ " (the 45 factors relating to the nature, range, shape, and location of the lesions) showed significant differences in the constituent ratios for lesion location (the superior lobe/inferior lobe of the left lung), lesion shape (wedge shape), lesions near the interlobular pleura (interlobular septal thickening, GGO associated with interlobular pleura traction and displacement), vascular changes (blood vessel thickening and vascular convergence sign), and banyan crown sign according to the clinical classification $(\mathrm{P}<0.05)$ (Table S5).

\section{ROC curve analysis of CT findings for the diagnosis of COVID-19}

The following 6 CT imaging examination indexes were selected mainly from the 109 patients on admission: the shape of the lesions (round), the location of the lesions (R9 lateral basal segment of the inferior lobe of the right lung), halo sign $(-/+)$, lesions near the chest wall [with or without subpleural lucency shadow $(-/+)$ ], lesion location (L3 anterior segment of the superior lobe of the left lung), and lesions near the chest wall [interlobular septal thickening $(-/+)]$. The ROC curve was generated, and COVID-19 diagnostic model 1 was constructed (Table S6, Figure 10), achieving a maximum AUC value of 0.8016 [95\% confidence interval (CI): 0.6759-0.9274].

Based on COVID-19 diagnostic model 1, a second model, COVID-19 diagnostic model 2 (Table S4, Figure 11), was constructed by adding 5 more CT imaging indexes, including the location of the lesions (L6 dorsal segment of the inferior lobe of the left lung and R8 anterior basal 

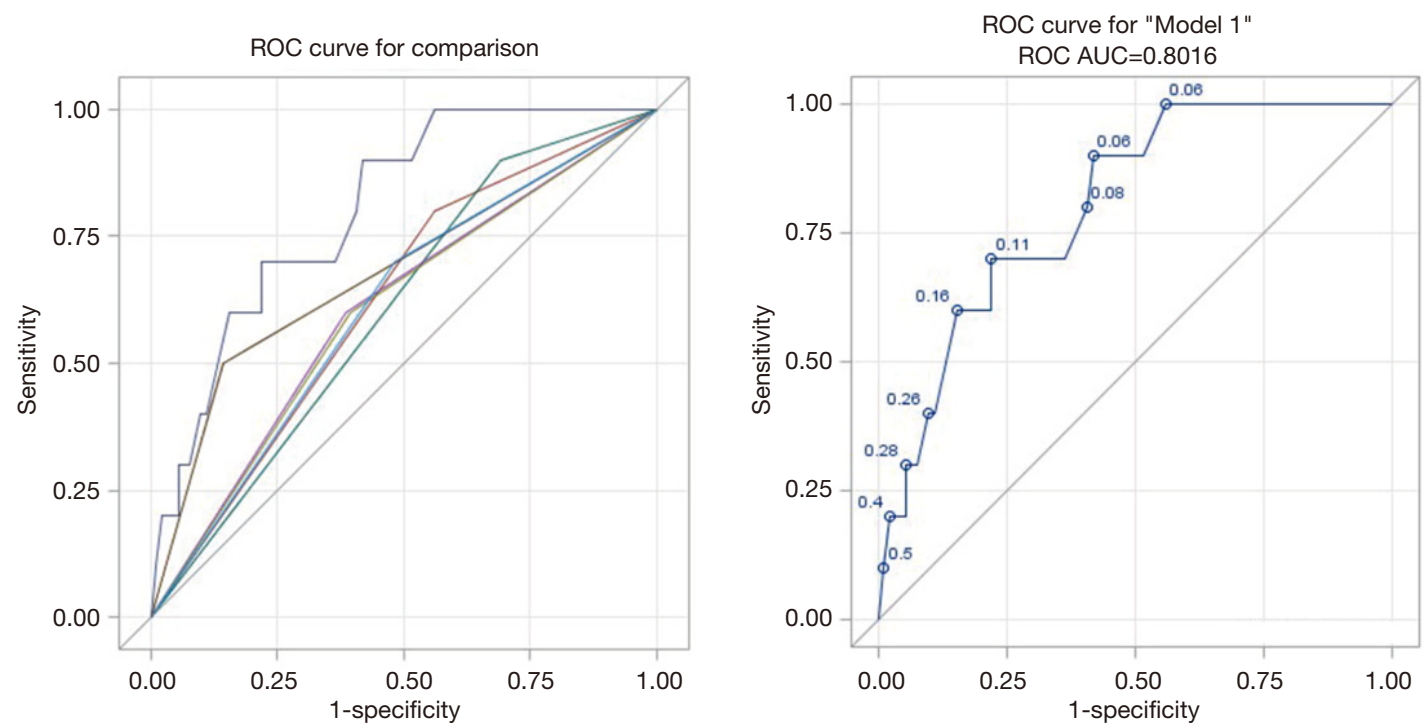

Figure 10 Receiver operating characteristic (ROC) curve of the 6 indexes for the diagnosis of COVID-19, with an area under the ROC curve (AUC) of 0.8016. COVID-19, coronavirus disease 2019.
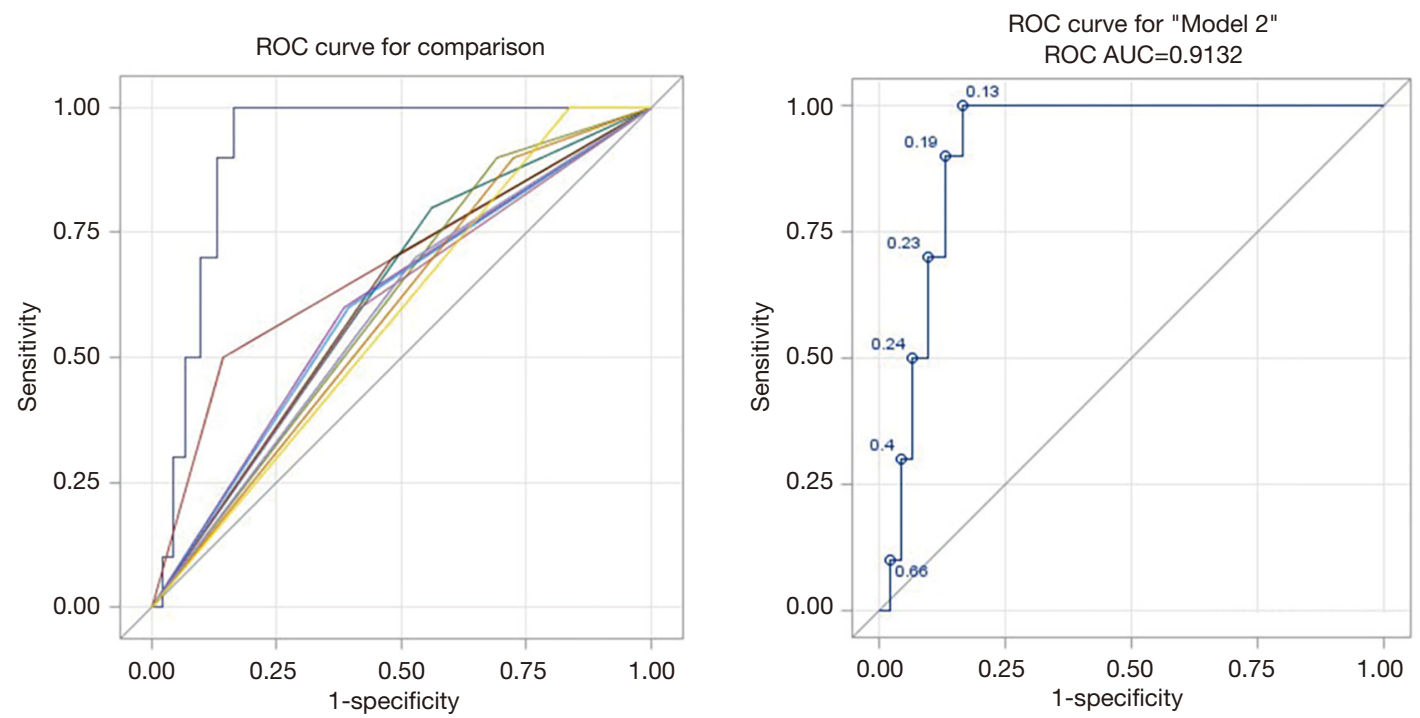

Figure 11 Receiver operating characteristic (ROC) curve of the 11 indexes for the diagnosis of COVID-19, with an area under the ROC curve (AUC) of 0.9132. COVID-19, coronavirus disease 2019.

segment of the inferior lobe of the right lung), the nature of the lesions (mixed and pure consolidation lesions), and the shape of the lesions (wedge-shaped). The ROC curve was generated, and the model yielded a maximum AUC value of 0.9132 (95\% CI: 0.8571-0.9693).

Similarly, based on COVID-19 diagnostic model 2, a third model, COVID-19 diagnostic model 3 (Table S4,
Figure 12), was constructed through the additional of 3 more CT imaging examination indexes, including the lesion location (L4 superior lingular segment of the superior lobe of the left lung and R1 apical segment of the superior lobe of the right lung) and the lesion range (near the lung hilus [around the segmental and lobar bronchus]). The ROC curve was generated, and the model achieved a maximum 

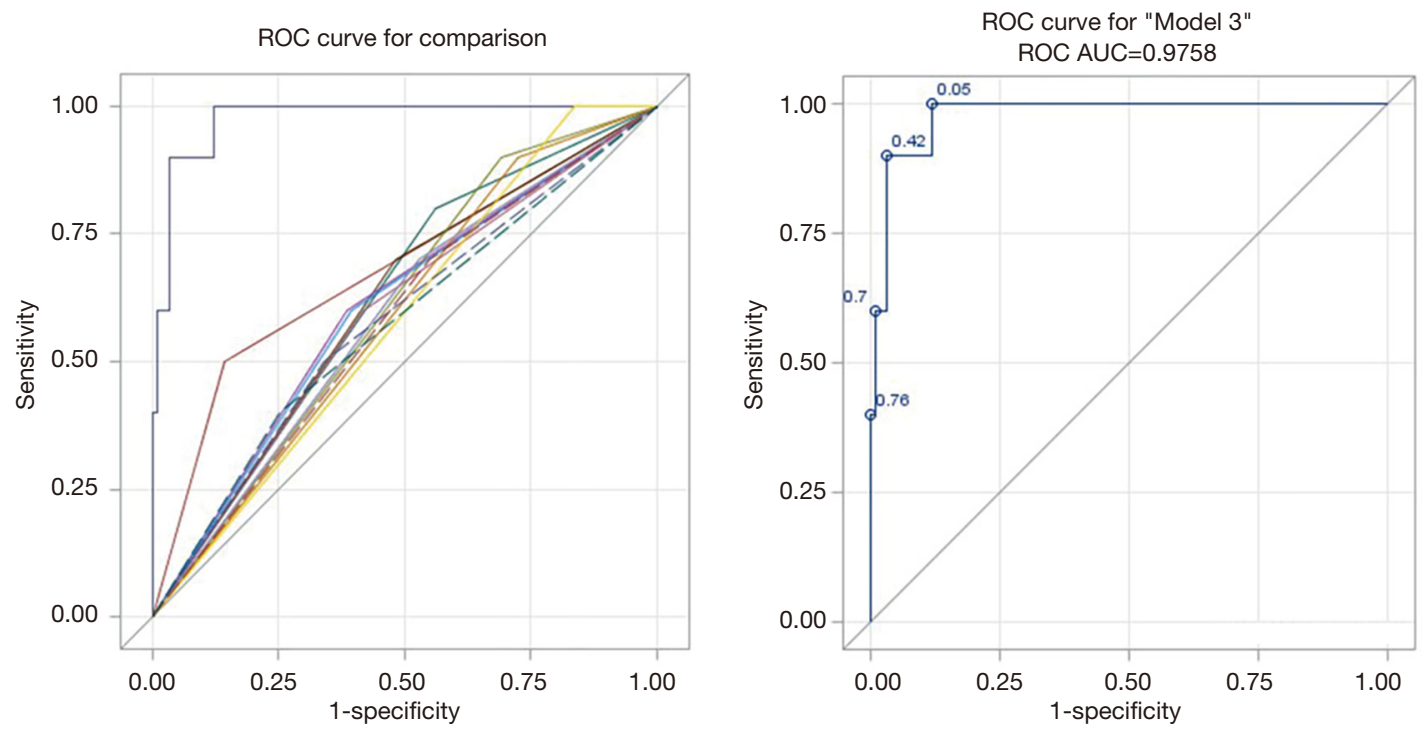

Figure 12 Receiver operating characteristic (ROC) curve of the 14 indexes for the diagnosis of COVID-19, with an area under the ROC curve (AUC) of 0.9758. COVID-19, coronavirus disease 2019.

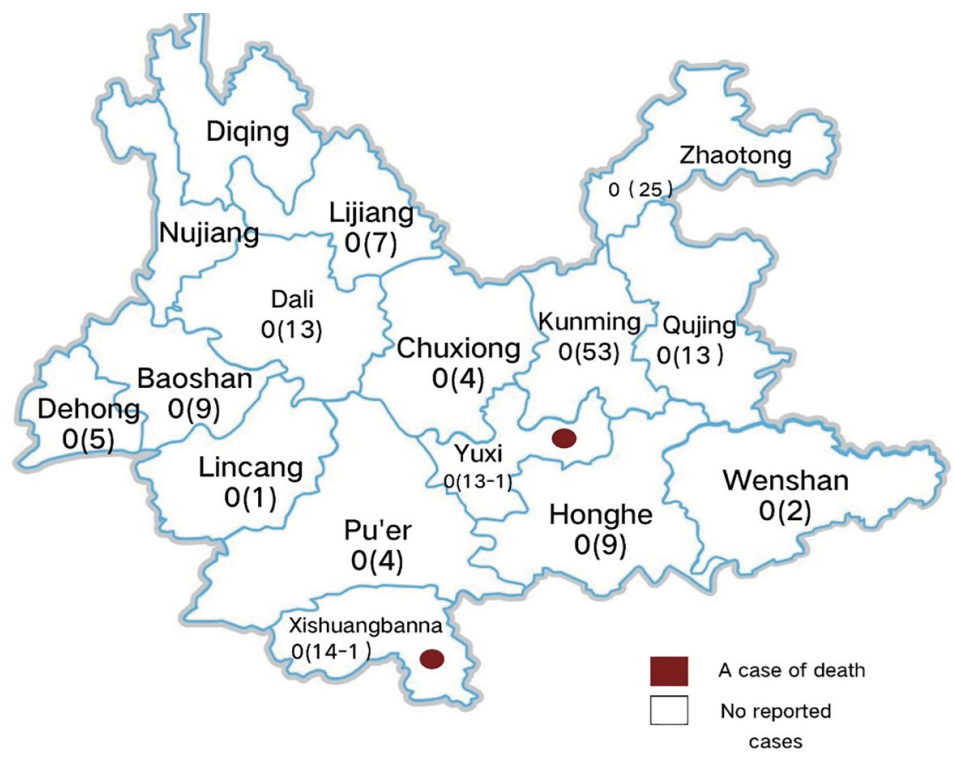

Figure 13 Geographic distribution of COVID-19 cases in Yunnan as of March 14, 2020. The number of confirmed cases hospitalized for treatment across the 16 cities and prefectures of Yunnan province has since decreased to zero. The numbers outside the brackets indicate the cumulative number of confirmed cases, and the numbers in the brackets indicate the cumulative number of cured cases. The cumulative number of confirmed cases in each prefecture and city are as follows: 53 cases in Kunming (all cured and discharged), 25 cases in Zhaotong (all cured and discharged), 15 cases in Dai Autonomous Prefecture of Xishuangbanna (14 cured and 1 died), 14 cases in Yuxi (13 cured and 1 died), 13 cases in Qujing (13 cured and discharged), 13 cases in Dali Bai Autonomous Prefecture (all cured and discharged), 9 cases in Baoshan (all cured and discharged), 9 cases in Hani-Yi Autonomous Prefecture of Honghe (all cured and discharged), 7 cases in Lijiang (all cured and discharged), 5 cases in Dehong Autonomous Prefecture (all cured and discharged), 4 cases in Pu'er (all cured and discharged), 4 cases in Chuxiong Prefecture (all cured and discharged), 2 cases in Wenshan Zhuang and Miao Autonomous Prefecture (both cured and discharged), and 1 case in Lincang (cured and discharged). COVID-19, coronavirus disease 2019. 
AUC value of 0.9758 (95\% CI: 0.9466-1).

\section{Discussion}

COVID-19 is caused by the infection of $\beta$-genus 2019$\mathrm{nCoV}$, which is an enveloped, usually polymorphic RNA virus characterized by round or oval-shaped particles with a diameter of $60-140 \mathrm{~nm}$. Other human coronavirus species that cause respiratory tract infections include severe acute respiratory syndrome coronavirus (SARS-CoV) $(10,11)$, which caused the 2003 SARS epidemic in China, and Middle East respiratory syndrome coronavirus (MERS$\mathrm{CoV})(12,13)$, which caused the 2012 epidemic in the Middle East. Interestingly, studies have found that many patients with 2019-nCoV infection show clinical symptoms similar to those of SARS and MERS patients (14-16). However, although the $2019-\mathrm{nCoV}$ is more infectious than both SARS and MERS, it has a lower mortality rate (17-21). A previous study showed that the similarity in gene sequences between 2019-nCoV and SARS-CoV and MERS-CoV was $79 \%$ and $50 \%$, respectively (22). Several studies have confirmed that SARS-CoV and MERS-CoV both originated from bats and were directly transmitted to humans from the masked palm civet and single-humped camel, respectively. However, further investigation is still needed to confirm the origin of 2019-nCoV (23-25).

Since December 2019, COVID-19 has spread throughout all over the world. After confirmation of the first imported case of COVID-19 in Yunnan on January 21,2020 , the epidemic gradually went on to affect all cities across the province (except Diqing Tibetan Autonomous Prefecture and Nujiang of the Lisu Autonomous Prefecture). As of 24:00 on March 14, 2020, Yunnan had confirmed 174 cases, among which 2 cases died, with the rest of the cases cured and discharged (see Figure 13 for a detailed case distribution). The present study included 143 confirmed cases, among which 34 with normal initial CT findings were excluded. After the baseline, clinical, and imaging data of the remaining 109 cases had been collected, COVID-19 chest CT diagnostic models were established, and their effectiveness and applicability were evaluated to provide a basis for the clinical diagnosis of COVID-19.

The changes in the conditions of COVID-19 patients are related to many factors, including constitution, basic diseases, and treatment plans. Relapse has been noted in a number of patients, even progressing to acute respiratory distress syndrome or multiple organ failure, which may be associated with the "inflammatory storm" in patients $(26,27)$.
The present study observed different sites, ranges, and degrees of lesion involvement in the lungs among patients at the initial stage of admission. Specifically, most cases had multiple lesions, and the involved sites were mainly concentrated in the inferior lobes of the lungs $57.8 \%$ in the posterior basal segment of the inferior lobe of the right lung and $54.13 \%$ in the posterior basal segment of the inferior lobe of the left lung). Due to its small particle size, 2019-nCoV mainly invades the bronchioles via the airway after inhalation, inducing bronchiolitis and peripheral inflammation, as well as further spread to the distal end of the lung tissues. One study found that the virus tended to function better in the blood capillary-rich periphery of the lungs, especially in the lower lungs (28). In the present study, most of the lung lesions observed were located in the subpleural area of the peripheral zone of the inferior lobes of both lungs, which was consistent with the distribution characteristics of the initial lesions.

The current study found that patients with COVID-19 mainly presented with pure GGO lesions (88.99\%), mixed GGO and consolidation lesions (46.79\%), and pure consolidation lesions (14.68\%) during CT examination. The presence of associated vascular thickening was observed in $79.82 \%$ of patients, and the lesions located near the interlobular pleura and the chest wall were observed in $57.8 \%$ and $87.16 \%$ of patients, respectively. Moreover, the main manifestations included interlobular septal thickening, interlobular (adjacent) pleural thickening, subpleural lucency shadow, and interlobular pleural traction and displacement. Initially, virus particles were mainly involved in the interstitium around the distal bronchioles below the lobular bronchioles, namely the centrilobular interstitium. Given that the lymphatic return in the centrilobular region is concentric, GGO lesions that were round in shape were more likely to form. Subsequently, the round-shaped GGO lesions gradually spread across the entire secondary pulmonary lobule and peripheral lung tissues, resulting in the formation of patchy GGO, with the sizes of lesions consistent with the secondary pulmonary lobule. Furthermore, with the enlargement and fusion of the lesions, large patchy GGOs formed. When the perilobular interstitium was involved, the GGOs were usually banded. Considering that the lymphatic return in this area is the drainage to the subpleural and peripheral region of the interlobular septa, the focus adhered to the pleura and spread along the interlobular septum on both sides, resulting in the long axis of the focus becoming parallel to the pleura. As such, all patients in our study 
exhibited mostly large, patchy, and banded GGO. When entering into the alveoli, $2019-\mathrm{nCoV}$ is fused to type II alveolar epithelial cells, causing a decrease in the secretion of alveolar surfactant and further resulting in adhesive atelectasis. The GGO lesions were classified as a consolidation shadow, which was consistent with the clinical symptoms, such as hypoxemia and dyspnea. This may also be the reason why a consolidation shadow was observed in $14.68 \%$ of the patients included in this study. Interlobular septal thickening often manifested as a short strip-like highdensity shadow extending to the pleura on CT, which is also known as a septal shadow. Studies have suggested that interlobular septal thickening can be observed in the early stages of COVID-19 and throughout the duration of the disease course $(12,13,28)$, indicating that COVID-19 has a tendency to involve the interstitium and cause fibrosis. Consistent with the aforementioned studies, our results showed that $27.52 \%$ and $56.88 \%$ of the patients exhibited interlobular septal thickening in lesions near the pleura and chest wall, respectively.

At present, COVID-19 is diagnosed through RTPCR of respiratory or blood samples, which carries some disadvantages, including the risk of false negative results and longer detection times. Meanwhile, chest CT as a certain role in addressing missed diagnoses caused by false negative results and other shortcomings of nucleic acid detection, provided it has a relatively high diagnostic efficiency. Through detailed statistical description and analysis of clinical data, epidemiological history, and chest CT imaging data from 109 patients, the present study found statistically significant differences in abnormal CT imaging signs among patients according to their epidemiological history and clinical classification, suggesting that abnormal chest CT imaging features had certain correlations with epidemiological history, lesion shape, location, range, and nature. In other words, abnormal CT imaging features could be used to further determine the shape, location, range, and nature of COVID-19 lesions. To further verify our findings, we initially identified 6 indexes reflecting abnormal CT imaging features and constructed COVID-19 diagnostic model 1, which had a maximum AUC value of 0.8016. Based on COVID-19 diagnostic model 1, 5 new indexes were added to establish COVID-19 diagnostic model 2, which had a maximum AUC value of 0.9132 . Finally, after the addition of 3 new indexes to COVID-19 diagnostic model 2, the final model utilized, COVID-19 diagnostic model 3, was constructed. COVID-19 diagnostic model 3 achieved a maximum AUC value of 0.9758 , indicating it to have the highest diagnostic efficiency among the 3 models, and it played an important role in the clinical diagnosis of COVID-19. Additionally, our findings showed significant differences in abnormal CT imaging signs (+/-) among patients with different epidemiological histories. Furthermore, stratified analysis of abnormal CT imaging signs (+/-) showed significant differences between firstand second-generation patients in terms of lesion location, lesion range, the Relationship between lesions and the chest wall or the interlobar pleura. Additionally, secondgeneration patients developed significantly fewer interstitial lesions in the lungs than first-generation patients, perhaps due to the gradual weakening of viral toxicity during the process of transmission, resulting in the occurrence of less obvious abnormal interstitial lesions, as well as the nonobvious CT imaging features specific to COVID-19.

This study has some limitations that need to be noted. First, due to geographical constraints, the current study had a relatively small sample size, and only a small number of severe and critical patients were included. Thus, future multi-center collaborative studies with large sample sizes are needed. Second, given that the study only summarized the initial CT examination findings, future studies need to include follow-up CT data of patients in the later stages of COVID-19 for summary and comprehensive analysis.

In conclusion, the present study showed that COVID-19 chest CT diagnostic model 3 had a relatively high efficiency for the diagnosis of abnormal lung lesions in patients with COVID-19. This diagnostic model has significant applicability and may provide a reference for the clinical diagnosis, evaluation of curative effect, and prognosis among patients with COVID-19 in the future.

\section{Acknowledgments}

We thank all participants of this project. Funding: None.

\section{Footnote}

Reporting Checklist: The authors have completed the MDAR checklist. Available at http://dx.doi.org/10.21037/apm-202603

Data Sharing Statement: Available at http://dx.doi. org/10.21037/apm-20-2603

Conflicts of Interest: All authors have completed the ICMJE 
uniform disclosure form (available at http://dx.doi. org/10.21037/apm-20-2603). The authors have no conflicts of interest to declare.

Ethical Statement: The authors are accountable for all aspects of the work in ensuring that questions related to the accuracy or integrity of any part of the work are appropriately investigated and resolved. All procedures performed in this study involving human participants were in accordance with the Declaration of Helsinki (as revised in 2013). This retrospective study was approved by the institutional review board of the First Affiliated Hospital of Kunming Medical University, Kunming Medical University, Kunming, China. Because of the retrospective nature of the research, the requirement for written informed consent was waived.

Open Access Statement: This is an Open Access article distributed in accordance with the Creative Commons Attribution-NonCommercial-NoDerivs 4.0 International License (CC BY-NC-ND 4.0), which permits the noncommercial replication and distribution of the article with the strict proviso that no changes or edits are made and the original work is properly cited (including links to both the formal publication through the relevant DOI and the license). See: https://creativecommons.org/licenses/by-nc-nd/4.0/.

\section{References}

1. Li Q, Guan X, Wu P, et al. Early transmission dynamics in Wuhan, China, of novel coronavirus-infected pneumonia. N Engl J Med 2020;382:1199-207.

2. Rothe C, Schunk M, Sothmann P, et al. Transmission of 2019-nCoV Infection from an Asymptomatic Contact in Germany. N Engl J Med 2020;382:970-1.

3. National Health Commission of the People's Republic of China. Diagnosis and treatment protocol for novel coronavirus pneumonia (5th trial version) Available online: http://www.chinacdc.cn/jkzt/crb/xcrxjb/202002/ W020200205535799210271.pdf

4. Hui DS, Azhar EI, Madani TA, et al. The continuing 2019-nCoV epidemic threat of novel coronaviruses to global health -The latest 2019 novel coronavirus outbreak in Wuhan, China. Int J Infect Dis 2020;91:264-6.

5. Fang $\mathrm{Y}$, Zhang $\mathrm{H}, \mathrm{Xu} \mathrm{Y}$, et al. CT Manifestations of Two Cases of 2019 Novel Coronavirus (2019-nCoV) Pneumonia. Radiology 2020;295:208-9.

6. Ufuk F, Savas R. Chest CT features of the novel coronavirus disease (COVID-19). Turk J Med Sci 2020;50:664-78.

7. Wang T, Zeng $\mathrm{X}$, Zhou $\mathrm{H}$, et al. Design, validation, and clinical practice of standardized imaging diagnostic report for COVID-19. Zhong Nan Da Xue Xue Bao Yi Xue Ban 2020;45:229-35.

8. Zhong Z, Hu Y, Yu Q, et al. Multistage CT features of coronavirus disease 2019. Zhong Nan Da Xue Xue Bao Yi Xue Ban 2020;45:250-6.

9. Ye Z, Zhang Y, Wang Y, et al. Chest CT manifestations of new coronavirus disease 2019 (COVID-19): a pictorial review. Eur Radiol 2020;30:4381-9.

10. Hu B, Zeng LP, Yang XL, et al. Discovery of a rich gene pool of bat SARS-related coronaviruses provides new insights into the origin of SARS coronavirus. PLoS Pathog 2017;13:e1006698.

11. Song HD, Tu CC, Zhang GW, et al. Cross-host evolution of severe acute respiratory syndrome coronavirus in palm civet and human. Proc Natl Acad Sci U S A 2005;102:2430-5.

12. Haagmans BL, Dhahiry SHSA, Reusken C, et al. Middle East respiratory syndrome coronavirus in dromedary camels: An outbreak investigation. Lancet Infect Dis 2014;14:140-5.

13. Drosten C, Kellam P, Memish ZA. Evidence for Camelto-Human Transmission of MERS Coronavirus. N Engl J Med 2014;371:1359-60.

14. Hui DSC, Zumla A. Severe Acute Respiratory Syndrome: Historical, Epidemiologic, and Clinical Features. Infect Dis Clin North Am 2019;33:869-89.

15. Who Mers-Cov Research G. State of Knowledge and Data Gaps of Middle East Respiratory Syndrome Coronavirus (MERS-CoV) in Humans. PLoS Curr 2013;5:ecurrents.ou tbreaks.0bf719e352e 7478f8ad85fa30127ddb8.

16. Lee JY, Kim YJ, Chung EH, et al. The clinical and virological features of the first imported case causing MERS-CoV outbreak in South Korea,2015. BMC Infect Dis 2017;17:498.

17. Huang C, Wang Y, Li X, et al. Clinical features of patients infected with 2019 novel coronavirus in Wuhan, China. Lancet 2020;395:497-506.

18. Yin Y, Wunderink RG. MERS, SARS and other coronaviruses as causes of pneumonia. Respirology 2018;23:130-7.

19. Song $Z$, Xu Y, Bao L, et al. From SARS to MERS, Thrusting Coronaviruses into the Spotlight. Viruses 2019;11:59.

20. Yang Y, Lu QB, Liu MJ, et al. Epidemiological and clinical 
features of the 2019 novel coronavirus outbreak in China. medRxiv 2020.

21. Chan-Yeung M, Rui-Heng XU. SARS: epidemiology. Respirology 2003;8:S9-14.

22. Zhu N, Zhang DY,Wang WL, et al. A Novel Coronavirus from Patients with Pneumonia in China, N Engl J Med 2020;382:727-33.

23. Tao Y, Shi M, Chommanard C, et al. Surveillance of Bat Coronaviruses in Kenya Identifies Relatives of Human Coronaviruses NL63 and 229E and Their Recombination History. J Virol 2017;91:e01953-16.

24. Cui J, Li F, Shi ZL. Origin and evolution of pathogenic coronaviruses. Nat Rev Microbiol 2019;17:181-92.

25. Zhou P, Fan H, Lan T, et al. Fatal swine acute diarrhoea

Cite this article as: Li X, Yuan F, Zhang Z, Yang J, Zhang J, Li Z, Peng Y, Jiang Y, Yi W, Ma J, Zhao W, He B, Wu L, Wang K. Clinical utility of a computed tomography-based receiver operating characteristic curve model for the diagnosis of COVID-19. Ann Palliat Med 2021;10(2):2048-2061. doi: 10.21037/ apm-20-2603 syndrome caused by an HKU2-related coronavirus of bat origin. Nature 2018;556:255-8.

26. Gupta KK, Khan MA, Singh SK. Constitutive Inflammatory Cytokine Storm: A Major Threat to Human Health. J Interferon Cytokine Res 2020;40:19-23.

27. Xu Z, Shi L, Wang Y, et al. Pathological findings of COVID-19 associated with acute respiratory distress syndrome. Lancet Respir Med 2020;8:420-2.

28. Chung M, Bernheim A, Mei X, et al. CT Imaging Features of 2019 Novel Coronavirus (2019-nCoV). Radiology 2020;295:202-7.

(English Language Editor: J. Reynolds) 


\section{Supplementary}

Table S1 Baseline information of the 109 patients with COVID-19

\begin{tabular}{|c|c|}
\hline & Cases $(n=109)$ \\
\hline \multicolumn{2}{|l|}{ Age } \\
\hline Mean (SD) & $46(16.8 \%)$ \\
\hline Range & $3-78$ \\
\hline$\leq 29$ & $22(20.18 \%)$ \\
\hline$\leq 39$ & $16(14.68 \%)$ \\
\hline $40-49$ & $22(20.18 \%)$ \\
\hline $50-59$ & $18(16.51 \%)$ \\
\hline $60-69$ & $22(20.18 \%)$ \\
\hline$\geq 70$ & $9(8.26 \%)$ \\
\hline \multicolumn{2}{|l|}{ Sex } \\
\hline Male & $54(49.54 \%)$ \\
\hline Female & $55(50.46 \%)$ \\
\hline \multicolumn{2}{|l|}{ Occupation } \\
\hline Retiree & $21(19.27 \%)$ \\
\hline Office worker & $12(11.01 \%)$ \\
\hline Self-employed & $10(9.17 \%)$ \\
\hline Farmer & $9(8.26 \%)$ \\
\hline Student & $6(5.5 \%)$ \\
\hline Worker & $4(3.67 \%)$ \\
\hline Personnel in a specific technical field & $3(2.75 \%)$ \\
\hline Unemployed & $2(1.83 \%)$ \\
\hline Teacher & $2(1.83 \%)$ \\
\hline Free occupation & $1(0.92 \%)$ \\
\hline Tour guide & $1(0.92 \%)$ \\
\hline Doctor & $1(0.92 \%)$ \\
\hline \multicolumn{2}{|l|}{ Native province } \\
\hline Hubei & $58(53.21 \%)$ \\
\hline Yunnan & $43(39.45 \%)$ \\
\hline Hunan & $3(2.75 \%)$ \\
\hline Anhui & $2(1.83 \%)$ \\
\hline Zhejiang & $1(0.92 \%)$ \\
\hline Guangdong & $1(0.92 \%)$ \\
\hline Jiangxi & $1(0.92 \%)$ \\
\hline
\end{tabular}

Table S1 (continued)
Table S1 (continued)

\begin{tabular}{lc}
\hline & Cases $(\mathrm{n}=109)$ \\
\hline History of exposure & \\
Traveled from Wuhan to Yunnan & $42(38.53 \%)$ \\
Returned to Yunnan from Wuhan & $25(22.94 \%)$ \\
History of close contact & $18(16.51 \%)$ \\
Self-drove from Wuhan to Yunnan & $9(8.26 \%)$ \\
Returned to Yunnan via Wuhan & $9(8.26 \%)$ \\
Returned to Yunnan from a business trip to & $1(0.92 \%)$ \\
Wuhan & $1(0.92 \%)$ \\
Traveled to Wuhan and returned to Yunnan & $4(3.67 \%)$ \\
Unclear history of exposure & \\
Epidemiological history & $84(77.06 \%)$ \\
First generation & $20(18.35 \%)$ \\
Second generation & $2(1.83 \%)$ \\
Third generation & $3(2.75 \%)$ \\
Unclear &
\end{tabular}




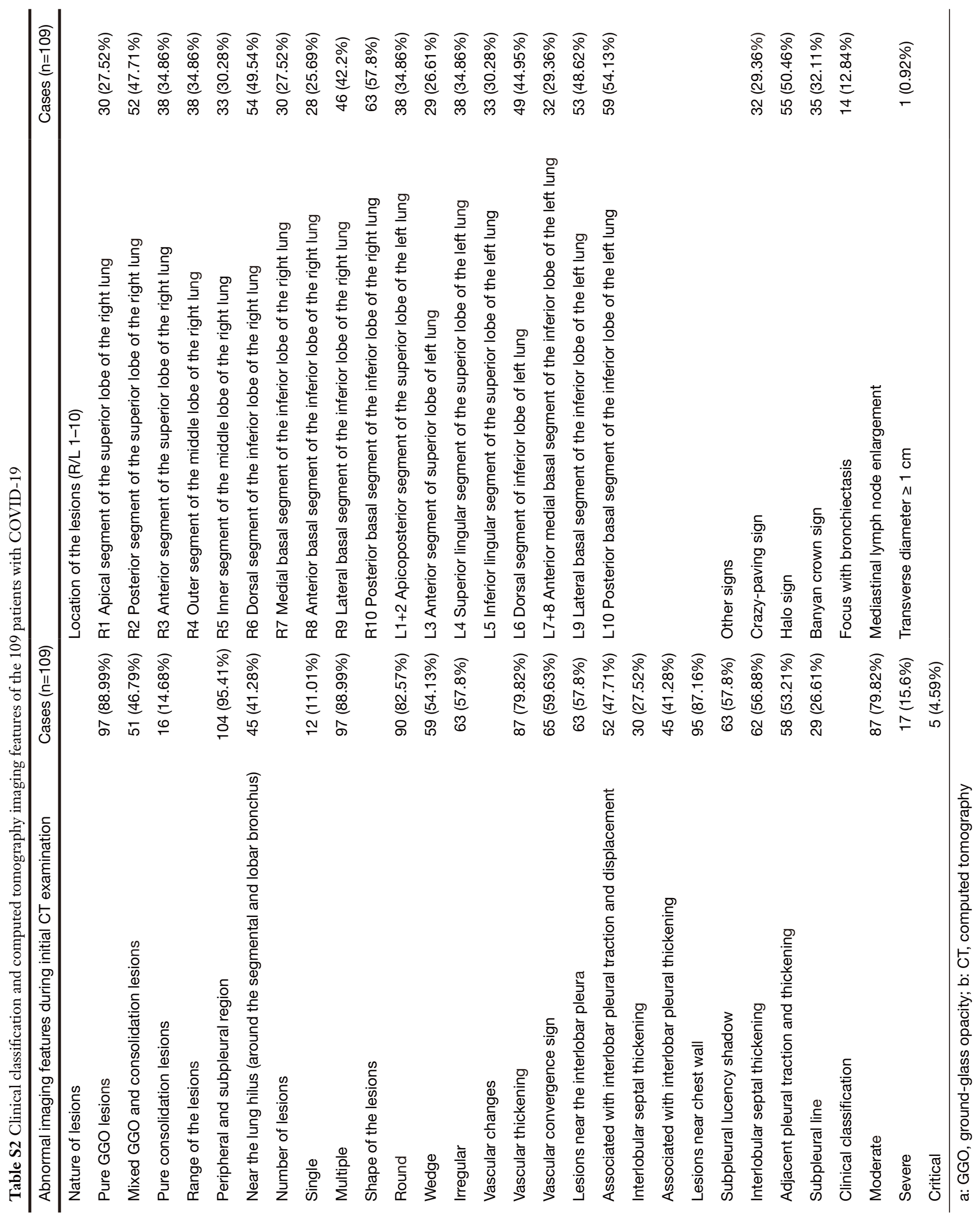


Table S3 Epidemiological history and computed tomography imaging features of the 109 patients with COVID-19

\begin{tabular}{|c|c|c|c|c|c|c|}
\hline \multirow{2}{*}{ CT imaging features } & \multicolumn{6}{|c|}{ Epidemiological history (Generations 1-3) } \\
\hline & $(+)$ & $(-)$ & $\chi^{2}$ & $\mathrm{P}$ & $\begin{array}{l}\text { Coefficient of } \\
\text { contingency }\end{array}$ & Cramer V \\
\hline \multicolumn{7}{|l|}{ Location of the lesions } \\
\hline $\begin{array}{l}\text { R7 Medial basal segment of the } \\
\text { inferior lobe of the right lung* }\end{array}$ & $30(28.04 \%)$ & $77(71.96 \%)$ & 9.0545 & 0.0108 & 0.2793 & 0.2909 \\
\hline Lobular septal thickening* & $61(57.01 \%)$ & $46(42.99 \%)$ & 8.7841 & 0.0124 & 0.2754 & 0.2865 \\
\hline $\begin{array}{l}\text { GGO associated with interlobar } \\
\text { pleural traction and displacement* }\end{array}$ & $52(48.6 \%)$ & $55(51.4 \%)$ & 8.1127 & 0.0173 & 0.2655 & 0.2754 \\
\hline Lesions near the interlobar pleura* & $63(58.88 \%)$ & $44(41.12 \%)$ & 8.0711 & 0.0177 & 0.2648 & 0.2746 \\
\hline
\end{tabular}

a: GGO, ground-glass opacity; b: CT, computed tomography; c: * $\mathrm{P}<0.05$

Table S4 Comparison of CT features between Generation 1 and Generation 2

Epidemiological history (Generations 1 and 2)

CT imaging features

\begin{tabular}{ccccccc}
\hline \multicolumn{3}{c}{ Accumulated lesions } & & \\
\hline$(+)$ & RR value $(+)$ & $(-)$ & RR value $(-)$ & & G Gamma
\end{tabular}

Location of the lesions

L3 Anterior segment of the superior lobe of the left lung

$\begin{array}{ccccccc}29(27.1 \%) & 2.373 & 78(72.9 \%) & 0.794 & 2.9311 & 0.0869 & -0.4986 \\ 38(35.51 \%) & 2.3274 & 69(64.49 \%) & 0.7206 & 4.2016 & 0.0404 & -0.5272\end{array}$

L4 Superior lingular segment of the superior lobe of the left lung*

Range of the lesions

Near the lung hilus (around the segmental and lobar $\begin{array}{lllllll}44(41.12 \%) & 1.6429 & 63(58.88 \%) & 0.5867 & 4.7191 & 0.0298 & 0.4737\end{array}$ bronchus) ${ }^{*}$

Lesions near chest wall

Interlobular septal thickening between the lesions and

$61(57.01 \%) \quad 2.1122$

$46(42.99 \%) \quad 0.5134$

$\begin{array}{lll}8.442 & 0.0037 & 0.6089\end{array}$

pleura*

Near the interlobar pleural lesion*

$63(58.88 \%) \quad 1.6429 \quad 44(41.12 \%) \quad 0.5867 \quad 4.7191 \quad 0.0298 \quad 0.4737$

Associated with interlobar pleural thickening*

$\begin{array}{lllllll}45(42.06 \%) & 2.1905 & 62(57.94 \%) & 0.6693 & 4.9626 & 0.0259 & 0.5319\end{array}$

Interlobar pleural GGO associated with

$\begin{array}{lllllll}52(48.6 \%) & 2.4643 & 55(51.4 \%) & 0.7804 & 3.8692 & 0.0492 & 0.4502\end{array}$

interlobar pleural traction and displacement*

$\begin{array}{lllllll}30(28.04 \%) & 2.4643 & 77(71.96 \%) & 0.7804 & 3.2645 & 0.0708 & 0.519\end{array}$

Interlobular septal thickening between
interlobar pleural lesions and interlobar pleura

GGO, ground-glass opacity; CT, computed tomography; RR, Relative risk; * $\mathrm{P}<0.05$ 
Table S5 Comparison of CT imaging characteristics between the clinical classifications

\begin{tabular}{|c|c|c|c|c|c|c|}
\hline \multirow{3}{*}{ CT imaging features } & \multicolumn{6}{|c|}{ Clinical classification (moderate, severe, and critical) } \\
\hline & \multicolumn{2}{|c|}{ Accumulated lesions } & \multirow{2}{*}{$\chi^{2}$} & \multirow{2}{*}{$\mathrm{P}$} & \multirow{2}{*}{$\begin{array}{l}\text { Coefficient of } \\
\text { contingency }\end{array}$} & \multirow{2}{*}{$\begin{array}{l}\text { Cramer } \\
\mathrm{V}\end{array}$} \\
\hline & $(+)$ & $(-)$ & & & & \\
\hline \multicolumn{7}{|l|}{ Location of the lesions } \\
\hline L1+2 Apicoposterior segment of the superior lobe of the left lung & $71(65.14 \%)$ & $38(34.86 \%)$ & 13.666 & 0.0011 & 0.3338 & 0.3541 \\
\hline L4 Superior lingular segment of the superior lobe of the left lung & $38(34.86 \%)$ & $71(65.14 \%)$ & 17.8642 & 0.0001 & 0.3753 & 0.4048 \\
\hline L5 Inferior lingular segment of the superior lobe of the left lung & $33(30.28 \%)$ & $76(69.72 \%)$ & 11.9593 & 0.0025 & 0.3144 & 0.3312 \\
\hline L6 Dorsal segment of the inferior lobe of the left lung & 49 (44.95\%) & $60(55.05 \%)$ & 6.1847 & 0.0454 & 0.2317 & 0.2382 \\
\hline L10 Posterior basal segment of the inferior lobe of the left lung & $59(54.13 \%)$ & $50(45.87 \%)$ & 9.3728 & 0.0092 & 0.2814 & 0.2932 \\
\hline R3 Anterior segment of the superior lobe of the right lung & $38(34.86 \%)$ & $71(65.14 \%)$ & 7.1275 & 0.0283 & 0.2477 & 0.2557 \\
\hline R4 Outer segment of the middle lobe of the right lung & $71(65.14 \%)$ & $38(34.86 \%)$ & 13.8732 & 0.001 & 0.336 & 0.3568 \\
\hline R7 Medial basal segment of the inferior lobe of the right lung & $30(27.52 \%)$ & $79(72.48 \%)$ & 4.5395 & 0.1033 & 0.2 & 0.2041 \\
\hline Shape of the lesions (wedge-shaped) & $59(54.13 \%)$ & $50(45.87 \%)$ & 19.0126 & $<.0001$ & 0.3854 & 0.4176 \\
\hline Lesions near the interlobar pleura & $63(57.8 \%)$ & $46(42.2 \%)$ & 12.7044 & 0.0017 & 0.3231 & 0.3414 \\
\hline $\begin{array}{l}\text { GGO lesions near interlobar pleura associated with interlobar pleural } \\
\text { traction and displacement }\end{array}$ & $52(47.71 \%)$ & 57 (52.29\%) & 9.6776 & 0.0079 & 0.2856 & 0.298 \\
\hline Interlobular septal thickening & $30(27.52 \%)$ & $79(72.48 \%)$ & 7.3053 & 0.0259 & 0.2506 & 0.2589 \\
\hline \multicolumn{7}{|l|}{ Other signs } \\
\hline Banyan crown sign & $35(32.11 \%)$ & $74(67.89 \%)$ & 12.6062 & 0.0018 & 0.322 & 0.3401 \\
\hline Crazy-paving sign & $32(29.36 \%)$ & $77(70.64 \%)$ & 5.4652 & 0.065 & 0.2185 & 0.2239 \\
\hline Enlarged lymph nodes in the mediastinum (transverse diameter $\geq 1 \mathrm{~cm}$ ) & $1(0.92 \%)$ & $108(99.08 \%)$ & 5.4619 & 0.0652 & 0.2184 & 0.2239 \\
\hline Lesions associated with bronchiectasis & $14(12.84 \%)$ & $95(87.16 \%)$ & 5.4318 & 0.0661 & 0.2179 & 0.2232 \\
\hline
\end{tabular}

GGO, Ground-glass opacity; CT, computed tomography; *, P $<0.05$ 
Table S6 ROC model (COVID-19)

\begin{tabular}{|c|c|c|c|c|c|c|}
\hline ROC correlation model/statistics & Area & $\begin{array}{l}\text { Standard } \\
\text { deviation }\end{array}$ & $\begin{array}{l}\text { Wald 95\% } \\
\text { confidence } \\
\text { interval }\end{array}$ & Somers' D & Gamma & Tau-a \\
\hline ROC model 2 & 0.9132 & 0.0286 & $0.8571-0.9693$ & 0.8264 & 0.8264 & 0.1489 \\
\hline ROC model 3 & 0.9758 & 0.0149 & $0.9466-1$ & 0.9516 & 0.9516 & 0.1715 \\
\hline Shape of the lesions (round-shaped) & 0.6786 & 0.0853 & $0.5113-0.8459$ & 0.3571 & 0.7143 & 0.0644 \\
\hline \multicolumn{7}{|l|}{ Location of the lesions } \\
\hline R9 Lateral basal segment of the inferior lobe of the right lung & 0.6198 & 0.0716 & $0.4794-0.7601$ & 0.2396 & 0.5166 & 0.0432 \\
\hline L3 Anterior segment of the superior lobe of the left lung & 0.6038 & 0.0556 & $0.4949-0.7128$ & 0.2077 & 0.6 & 0.0374 \\
\hline L4 Superior lingular segment of the superior lobe of the left lung & 0.5797 & 0.087 & $0.4092-0.7502$ & 0.1593 & 0.3187 & 0.0287 \\
\hline R1 Apical segment of the superior lobe of the right lung & 0.5736 & 0.0848 & $0.4074-0.7398$ & 0.1473 & 0.3268 & 0.0265 \\
\hline Halo sign & 0.6082 & 0.0808 & $0.4499-0.7666$ & 0.2165 & 0.4273 & 0.039 \\
\hline \multicolumn{7}{|l|}{ Lesions near the chest wall } \\
\hline Subpleural lucency shadow & 0.6077 & 0.0856 & $0.44-0.7754$ & 0.2154 & 0.4118 & 0.0388 \\
\hline Interlobular septal thickening & 0.6022 & 0.0856 & $0.4344-0.77$ & 0.2044 & 0.3924 & 0.0368 \\
\hline Shape of the lesions (wedge-shaped) & 0.5863 & 0.0808 & $0.4279-0.7446$ & 0.1725 & 0.3528 & 0.0311 \\
\hline
\end{tabular}

a: GGO, Ground-glass opacity; b: ROC, receiver operating characteristic. 